\title{
Persistence of fluoroquinolone-resistant Salmonella enterica serovar Kentucky from poultry and poultry sources in Nigeria
}

\author{
Ibrahim A. Raufu ${ }^{1}$, Kayode Fashae ${ }^{2}$, James A. Ameh ${ }^{3}$, AbdulGaniyu Ambali ${ }^{1}$, Folashade T. Ogunsola ${ }^{4}$, \\ Akitoye O. Coker ${ }^{4}$, Rene S. Hendriksen ${ }^{5}$ \\ ${ }^{1}$ Department of Veterinary Microbiology, Faculty of Veterinary Medicine, University of Ilorin, Ilorin, Nigeria \\ ${ }^{2}$ Department of Microbiology, University of Ibadan, Ibadan, Nigeria \\ ${ }^{3}$ Department of Veterinary Microbiology, Faculty of Veterinary Medicine, University of Maiduguri, Maiduguri, Nigeria \\ ${ }^{4}$ Department of Medical Microbiology and Parasitology, College of Medicine, University of Lagos, Idi-Araba, Lagos, \\ Nigeria \\ ${ }^{5}$ WHO Collaborating Center for Antimicrobial Resistance in Food borne Pathogens and European Union Reference \\ Laboratory for Antimicrobial Resistance, National Food Institute, Technical University of Denmark, Kgs. Lyngby, \\ Denmark
}

\begin{abstract}
Introduction: This study investigated the antimicrobial resistance and clonality of Salmonella enterica serotype Kentucky in poultry and poultry sources in Nigeria, and compared the isolates with the clone of $S$. Kentucky STI98-X1 CIP ${ }^{\mathrm{R}}$ using (PFGE) and (MIC).

Methodology: Fecal samples from chickens and poultry sources (litter, water, rodent and lizard fecal samples) were collected from fourteen (14) poultry farms in 2007, 2010 and 2011 and were analyzed for $S$. Kentucky.

Results and conclusions: Six percent of the samples were positive for $S$. Kentucky - all resistant to nalidixic acid and ciprofloxacin. The isolates are grouped within the PFGE cluster X1 of $S$. Kentucky STI98 CIP ${ }^{\mathrm{R}}$, indicating the association to the emerging and widely spread $\mathrm{CIP}^{\mathrm{R}} S$. Kentucky clone with poultry and poultry sources.
\end{abstract}

Key words: Salmonella Kentucky; antimicrobial resistance; poultry; Ibadan; Maiduguri; Nigeria; MIC determination; PFGE; fluoroquinolones; ST198; ciprofloxacin resistance

J Infect Dev Ctries 2014; 8(3):384-388. doi:10.3855/jidc.3495

(Received 04 March 2013 - Accepted 17 June 2013)

Copyright $(2014$ Raufu et al. This is an open-access article distributed under the Creative Commons Attribution License, which permits unrestricted use, distribution, and reproduction in any medium, provided the original work is properly cited.

\section{Introduction}

Salmonella enterica serotype Kentucky $(S$. Kentucky) has been closely associated with poultry since it was first isolated in 1937 from a chick in the United States of America (USA) [1].

During the $1990 \mathrm{~s}$, a clone of ciprofloxacinresistant $\left(\mathrm{CIP}^{\mathrm{R}}\right) \mathrm{S}$. Kentucky $(\mathrm{MIC}>0.06 \mu \mathrm{g} / \mathrm{mL}$ according to EUCAST) of multilocus sequence type (MLST) ST198 containing pulsed-field gel electrophoresis (PFGE) pattern X1 emerged in Egypt, featuring high-level resistance to fluoroquinolones. Recently, the clone has spread to several countries, including sub-Saharan Africa, the Middle East, and Europe, causing infection in humans probably through contaminated imported foods or secondary contaminations [2].

Since poultry has been identified as a potential major vehicle for infection by this clone [3], a One Health surveillance approach is needed to detect, understand, and monitor the spread and persistency of this multidrug-resistant clone.

This study investigates the level of resistance and clonality of $S$. Kentucky in poultry and poultry sources over time in two geographical regions in Nigeria. Additionally, to compare the isolates with the clone of $S$. Kentucky STI98-X1 CIP ${ }^{\mathrm{R}}$ based on PFGE and minimum inhibition concentration (MIC) determination.

\section{Methodology}

Fecal and environmental samples were collected from poultry houses in western (Ibadan) and northeastern (Maiduguri) regions of Nigeria in the years 2007 and 2010 to 2011, respectively.

In Ibadan, a total of 641 samples from chickens were collected between March and May 2007 from nine different intensively managed farms, while in Maiduguri, five intensively managed poultry farms 
were visited between November 2010 and February 2011, and a total of 270 samples from chickens (feces) and poultry environment (litter, water, rodents, and lizards feces) were collected. Salmonella were presumptively identified using biochemical characterization according to the standard techniques recommended [4]. Full serotyping was carried out on all the presumptive isolates at the WHO reference laboratory in Thailand. MIC determination and PFGE were performed according to Hendriksen et al. [5]. The PFGE patterns were subsequently compared using Bionumeric software with the clone of $S$. Kentucky STI98-X1 CIP ${ }^{\mathrm{R}}[2]$. The antimicrobials and resistance cut-off values $(\mu \mathrm{g} / \mathrm{mL})$ were: AMP, ampicillin $(>8)$; AUG, amoxicillin-clavulanic acid $(\geq 32)$; APR, apramycin ( $>$ 32); XNL, ceftiofur $(>2)$; CHL, chloramphenicol ( $>16)$; CIP, ciprofloxacin (0.064-1; $>1$ ); COL, colistin (> 2); FFN, florfenicol (> 16); GEN, gentamicin $(>2)$; FOT, cefotaxime $(>0.5)$; NAL, nalidixic acid ( $>16)$; NEO, neomycin $(>4)$; SPE, spectinomycin (> 64); STR, streptomycin (> 16); SMX, sulfamethoxazole ( $>256)$; TET, tetracycline ( $>$ $8)$; TMP, trimethoprim $(>2)$. The MIC test was carried out on the isolates using a commercially prepared, dehydrated panel (Sensititre; TREK Diagnostic Systems Ltd., East Grinstead, England).

\section{Results}

Out of an overall 911 samples analyzed, 55 (6\%) were positive for $S$. Kentucky (44 and 11 isolates from Ibadan and Maiduguri respectively) . Forty-five of the isolates were from chicken fecal samples, while the remaining were distributed among samples from lizards $(n=3)$, water $(n=1)$, rodents $(n=5)$, and poultry house (litter; $\mathrm{n}=1$ ), as shown in Figure 1 .

Generally, all the isolates from the two geographic regions were resistant to nalidixic acid and ciprofloxacin (MIC $>4 \mu \mathrm{g} / \mathrm{mL}$ ); a full antibiogram is presented in Figure 1. In comparison to $S$. Kentucky from Maiduguri, the isolates from Ibadan exhibited the highest level of resistance, where isolate KS 13 from farm B conferred resistance to nine of all tested antimicrobials including ampicillin, ciprofloxacin, gentamicin, nalidixic acid, neomycin, spectomycin, streptomycin, sulfamethoxazole, and tetracycline. In addition, 28 isolates originating from nine different farms exhibited a similar resistance pattern, as KS 13 (excluding neomycin) conferred resistance to eight antimicrobials (Figure 1). Eleven isolates from four farms in Ibadan and 11 isolates from five poultry farms in Maiduguri shared the same resistance profile: ciprofloxacin, gentamicin, nalidixic acid, neomycin, spectomycin, streptomycin, sulfamethoxazole, and tetracycline.

None of the isolates were resistant to cefotaxime (FOT) and ceftiofur (XNL), indicating the absence of extended-spectrum cephalosporinases.

The 55 isolates were separated into four overall PFGE clades which consisted of $36,17,1$, and 1 isolates, respectively (Figure 1). Among the two largest clades, seven clusters of indistinguishable PFGE patterns were observed. The largest cluster consisted of 23 isolates from Ibadan (2007) and all isolates from Maiduguri (2010-2011) except for one. The isolates from Ibadan were all from chickens sampled from nine farms, whereas the isolates from Maiduguri all were from poultry feces and poultry environment of five intensively managed farms. Additionally, all isolates represented different antimicrobial resistant profiles (Figure

1). All the isolates were grouped within the PFGE cluster X1 of $S$. Kentucky STI98 CIP $^{\mathrm{R}}$ (data not shown) [2].

\section{Discussion}

This study investigated the presence of $\mathrm{CIP}^{\mathrm{R}} S$. Kentucky isolates from poultry and poultry sources over time from two regions in Nigeria.

We observed diversity based on PFGE, indicating the presence of several lineages in Nigeria. Despite the diversity, all samples still fell within the PFGE X1 strain, affirming their long-term presence in the study region [2].

Beyond quinolone resistance, additional resistance to streptomycin, spectinomycin, gentamicin, sulfamethoxazole, and tetracycline were observed in some $\mathrm{CIP}^{\mathrm{R}} S$. Kentucky isolates, which points to the presence of SGI $(9,10,11)$; further studies are required to confirm this speculation. The lack of effective policy to regulate the use of fluoroquinolones, including ciprofloxacin, enrofloxacin, and ofloxacin, in chicken production in Nigeria (unpublished data) may have contributed to the dissemination of multidrug-resistant non-typhoidal Salmonella; rapid spread has also been observed in Southeast Asia [2]. The emergence and spread of clinically significant clonal groups of Salmonella appear to occur on a regular basis [9]. 
Figure 1. Dendrogram showing the genotypic relatedness of the Salmonella enterica serovar Kentucky isolates from Nigeria based on $X b a$ I PFGE fingerprints

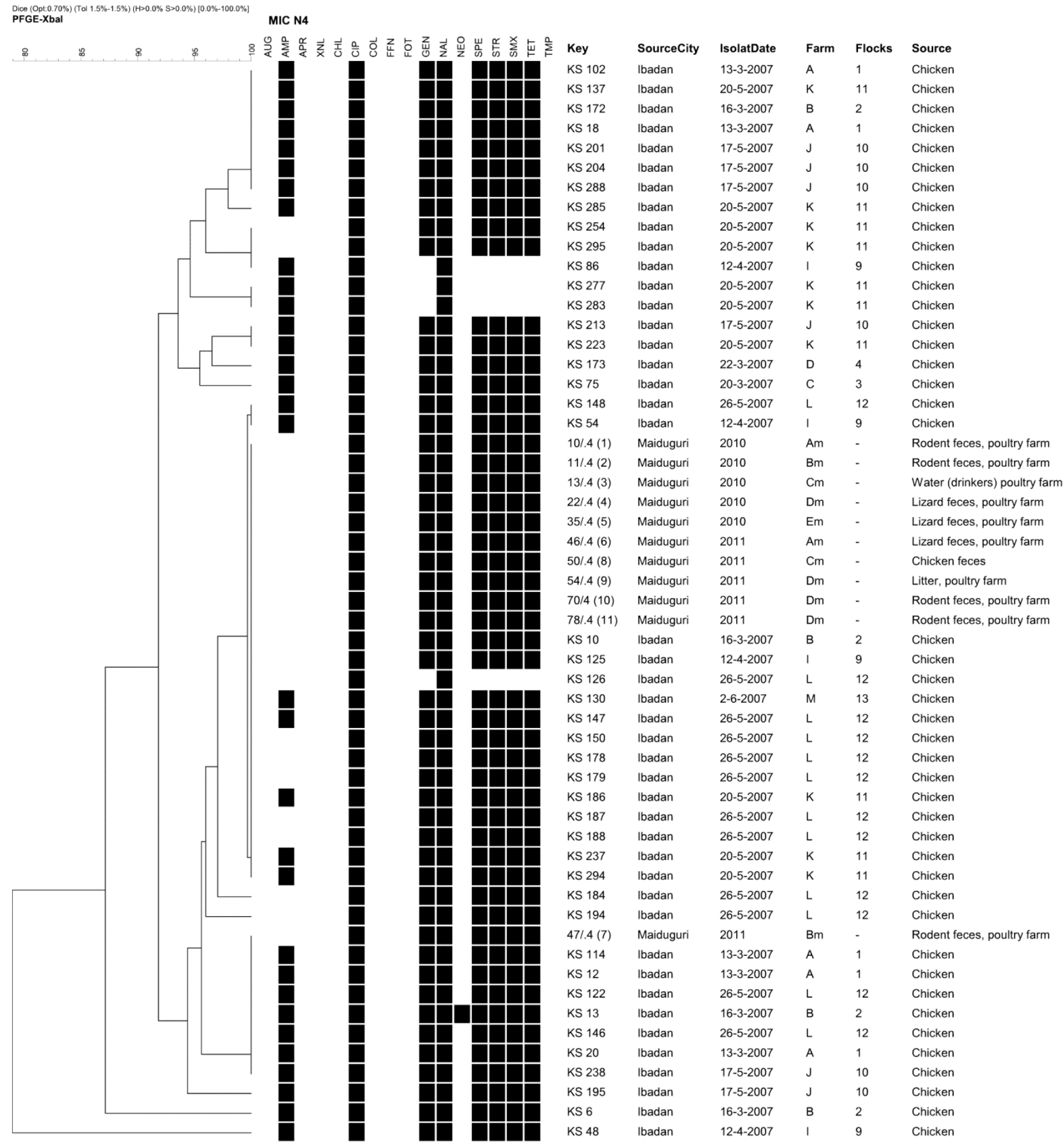

Black squares represent the isolates classified as resistant, Abbreviations: AMP, Ampicillin; AUG, amoxicillin + clavulanic acid; APR, apramycin; FOT, cefotaxime; XNL, ceftiofur; CHL, chloramphenicol; CIP, ciprofloxacin; COL, colistin; FFN, florfenicol; GEN, gentamicin; NAL, nalidixic acid; NEO, neomycin; SPE, spectinomycin; STR,streptomycin; SMX, sulfamethoxazole; TET, tetracycline; TMP, trimethoprim. 
This study documented a significant contamination of poultry with multidrug-resistant $S$. Kentucky, which reaffirmed the initial speculation of the existence of a prevailing circulating poultry-associated STI98-X1 $\mathrm{CIP}^{\mathrm{R}} S$. Kentucky in Africa, including Nigeria [2]. We speculate that one or more traits must be present in this serovar that underlie its success as colonizer of chickens; perhaps among these traits are those facilitating specific host-bacterial interactions and those that survive in modern poultry farm environments [10,11]. Further studies will be needed to confirm these hypotheses.

The extent of the emergence of the ST198-X1 $\mathrm{CIP}^{\mathrm{R}} S$. Kentucky clone in the poultry sector in different regions of Africa remains to be determined, but this preliminary investigation has revealed that poultry in Nigeria, where the industry uses indigenous domestic fowl, are largely contaminated with a single strain as defined by PFGE. This did not corroborate the hypothesis that the dissemination of a common contaminated poultry lineage throughout Africa might be responsible for the dissemination of this clone. More likely, it is contaminated food ingredients, including human food [12], that serve as a portal of entry into the agriculture production systems [13].

Practices such as the intensive (deep litter) and semi-intensive systems practised by most poultry establishments coupled with the unhygienic environment and poor management practices probably contribute to the spread and emergence of the clone and its widespread distribution and cross contamination of litter, reptiles, water, rodents, and lizards $[14,15]$.

\section{Conclusion}

Our study revealed the association of $\operatorname{CIP}^{\mathrm{R}} S$. Kentucky with poultry and the poultry environment. The lack of effective policy to control the use of antimicrobials in poultry in Nigeria may have contributed to its spread.

It is recommended that the authorities in Nigeria establish a National Salmonella Surveillance Program for effective long-term national and international joint integrated public health surveillance with surveillance of food animal populations for prompt identification and control of the epidemic non-typhoid Salmonella, including ST198-X1 $\mathrm{CIP}^{\mathrm{R}} S$. Kentucky clone; these would greatly increase the likelihood of early detection before the bacteria become widely disseminated. The data generated will enable policy makers to legislate on antimicrobial use in agriculture and clinical settings and the sub-therapeutic use of antimicrobials to promote growth and feeding efficiency in animals; this would lead to a reduction in antimicrobial resistance.

\section{Acknowledgements}

This study was supported in part by the University of Maiduguri fellowship for postgraduate training. Thanks to Bimbo Adesuyi and Adewale Bello from the Department of Microbiology, University of Ibadan, for assistance in sample collection. We are grateful to Chaiwat Pulsrikarn and Srirat Pornruangwong from the Department of Medical Sciences, WHO National Salmonella and Shigella Center, National Institute of Health, Ministry of Public Health, Thailand, for technical assistance in serotyping all the isolates

\section{References}

1. Edwards PR (1938) A new Salmonella type: Salmonella Kentucky. J. Hygiene (Lond) 38: 306-308.

2. Le Hello S, Hendriksen RS, Doublet B, Fisher I, Nielsen EM, Whichard JM, Bouchrif B, Fashae K, Granier SA, Silva NJ, Cloeckaert A, Threlfall EJ, Angulo FJ, Aarestrup FM, Wain J, Weill FX (2011) International Spread of an Epidemic Population of Salmonella enterica Serotype Kentucky ST198 Resistant to Ciprofloxacin. J. Infect Diseases 204: 675-684.

3. Johnson TJ, Thorsness JL, Anderson CP, Lynne AM, Foley SL, Han J, Fricke WF, McDermott PF, White DG, Khatri M, Stell AL, Flores C, Singer RS (2010) Horizontal gene transfer of a ColV plasmid has resulted in a dominant avian clonal type of Salmonella enterica serovar Kentucky. PLoS One 5: $1-10$.

4. Cowan ST and Steel KJ (1974) Manual for the Identification of Medical Bacteria, 2nd edition. London: Cambridge University Press 238p.

5. Hendriksen RS, Le Hello S, Bortolaia V, Pulsrikarn C, Møller NE, Pornruangmong S, Chaichana P, Svendsen AC, Weill FX, Aarestrup FM (2012) Characterization of Isolates of Salmonella enterica Serovar Stanley, a Serovar Endemic to Asia and Associated with Travel. J Clin Microbiol 50: 709720.

6. Butaye P, Michael GB, Schwarz S, Barrett TJ, Brisabois A, White DG (2006) The clonal spread of multidrug-resistant non-typhi Salmonella serotypes. Microbes Infect 8: 18911897.

7. Pedroso AA, Lee MD, Sellers H, Linneman E, Zamperini K, Maurer JJ (2007) Salmonella enterica Kentucky: a newly emergent poultry commensal adapted to life in the chicken cecum? Abstract presented at the 107th General Meeting of the American Society for Microbiology, Toronto, Canada, [Abst. 2030].

8. Joerger RD, Casey AS, Kalmia EK (2009) Comparison of Genetic and Physiological Properties of Salmonella enterica Isolates from Chickens Reveals One Major Difference Between Serovar Kentucky and Other Serovars: Response to Acid. Foodborne Pathog Dis 6: 503-512.

9. Levings RS, Partridge SR, Lightfoot D, Hall RM, Djordjevic SP (2005) New Integron associated gene cassette encoding a 3-N-aminoglycoside acetyltransferase. Antimicrob. Agents Chemother 49: 1238-1241. 
10. Levings RS, Partridge SR, Djordjevic SP, Hall RM (2007) SGI1-K, a variant of the SGI1 genomic island carrying a mercury resistance region, in Salmonella enterica serovar Kentucky. Antimicrob Agents Chemother 51: 317-323.

11. Le Hello S, Weill FX, Guibert V, Praud K, Cloeckaert A, Doublet B (2012) Early Strains of Multidrug-Resistant Salmonella enterica Serovar Kentucky Sequence Type 198 from Southeast Asia Harbor Salmonella Genomic Island 1-J Variants with a Novel Insertion Sequence. Antimicrob Agents Chemother 56: 5096-5102.

12. Centers for Disease Control and Prevention (2009) Multistate outbreak of Salmonella infections associated with peanut butter and peanut butter containing products-United States, 2008-2009. MMWR Morb Mortal Wkly Rep 58: 1-6.

13. Hedberg CW (2011) Challenges and Opportunities to Identifying and Controlling the International Spread of Salmonella. J Infect Diseases 204: 665-666.

14. Raufu I, Hendriksen RS, Ameh JA, Aarestrup FM (2009) Occurrence and characterization of Salmonella Hiduddify from chickens and poultry meat in Nigeria. Foodborne Pathog Dis 6: 425-430.

15. Waziri A, Raufu IA, Ambali A G,(2010) Poultry Management Errors among Farmers in Maiduguri Metropolitan Council and Jere Local Government Area, Maiduguri, Nigeria. Bull Anim Hlth Prod Afr 58: 385-387.

\section{Corresponding author}

Ibrahim Raufu, D.V.M., M.Sc., Ph.D.

Faculty of Veterinary Medicine

Department of Veterinary Microbiology

University of Ilorin

P.M.B. 1515, Nigeria

Phone: +2348027375931

Email: raufuib@yahoo.com

Conflict of interests: No conflict of interests is declared. 